\title{
Cordycepin inhibits the proliferation and progression of NPC by targeting the MAPK/ERK and $\beta$-catenin pathways
}

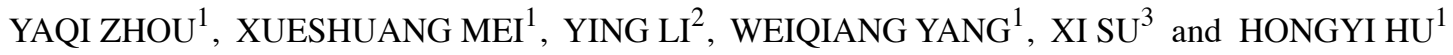 \\ Departments of ${ }^{1}$ Otorhinolaryngology, ${ }^{2}$ Radiology and ${ }^{3}$ Thyroid and Breast Surgery, \\ Peking University Shenzhen Hospital, Shenzhen Peking University-The Hong Kong University of \\ Science and Technology Medical Centre, Shenzhen, Guangdong 518000, P.R. China
}

Received July 13, 2021; Accepted August 23, 2021

DOI: $10.3892 / 01.2021 .13138$

\begin{abstract}
Cordycepin is an extract from the Cordyceps genus of ascomycete fungi. In the present study, the anticancer potential of cordycepin against nasopharyngeal carcinoma (NPC), and the potential underlying mechanisms, were investigated. Using Cell Counting Kit 8, wound-healing and Transwell assays, cordycepin was found to reduce the viability and inhibit the migration of C666-1 cells in a dose-dependent manner. In addition, in colony formation assays, co-treatment with cordycepin and cisplatin inhibited the proliferation of C666-1 cells. Furthermore, RNA sequencing analysis identified 72 significantly differentially expressed genes and different signaling pathways that may be regulated by cordycepin. After treatment with cordycepin, the expression levels of ERK1/2, phosphorylated ERK1/2 and $\beta$-catenin were significantly downregulated. Therefore, cordycepin may be a novel candidate for NPC treatment or a co-treatment candidate with cisplatin in chemotherapy.
\end{abstract}

\section{Introduction}

Nasopharyngeal carcinoma (NPC) is prevalent in East and Southeast Asia, including south China $(1,2)$. Due to the early onset of lymphatic metastasis, as well as a high recurrence rate, more efficient therapeutic methods are required $(1,3)$. Radiotherapy is currently the primary treatment type for non-metastatic NPC, which differs from advanced NPC $(3,4)$, which at present, is primarily treated with chemoradiotherapy

Correspondence to: Dr Hongyi Hu, Department of Otorhinolaryngology, Peking University Shenzhen Hospital, Shenzhen Peking University-The Hong Kong University of Science and Technology Medical Centre, 1120 Lianhua Road, Shenzhen, Guangdong 518000, P.R. China

E-mail: hyihu@pkuszh.com

Abbreviations: NPC, nasopharyngeal carcinoma; GO, gene ontology; KEGG, Kyoto Encyclopedia of Genes and Genomes; EGFR, epidermal growth factor receptor; NSCLC, non-small-cell lung cancer

Key words: NPC, cordycepin, migration, proliferation, ERK using a platinum-based reagent. Previous studies have shown that combination therapy of platinum with other drugs may significantly increase the efficacy of chemotherapy in advanced NPC. For example, a clinical trial has shown that the addition of gemcitabine and cisplatin-based induction chemotherapy to basic chemoradiotherapy significantly improved survival rates in locoregionally-advanced NPC (5). Other drugs have also been reported to enhance the efficacy of platinum-based chemotherapy in NPC (6-8). However, the incidence of adverse effects following combination treatment is still a concern.

Numerous studies have investigated natural products and compounds as novel treatments for different cancer types, or to enhance the efficacy of classic chemotherapeutic drugs such as cisplatin $(9,10)$. Cordycepin $(3$-deoxyadenosine) is a compound extracted from the Cordyceps genus of ascomycete fungi, which is used in traditional Chinese medicine (11). Previous studies have demonstrated the anticancer characteristics of cordycepin in different cancer cell types, including hepatocellular carcinoma, oral cancer and lung cancer (12-15); however, the underlying mechanisms remain unclear $(16,17)$.

The aim of the present study was to investigate the effects of cisplatin on NPC cells, (namely, whether it has the same effects as in other cancer types), using transcriptome sequencing to elucidate the underlying molecular mechanisms of cordycepin treatment in NPC.

\section{Materials and methods}

Reagents and cell culture. Cordycepin and cisplatin were purchased from MedChemExpress. Immediately before use, cordycepin was dissolved in media to generate a $10 \mathrm{mM}$ stock solution, and cisplatin was dissolved in $\mathrm{N}, \mathrm{N}$-dimethylformamide to generate a $10 \mathrm{mM}$ stock solution. Both stock solutions were stored at $-20^{\circ} \mathrm{C}$. The Human C666-1 NPC cell line, which is an EBV-positive NPC cell line taken from undifferentiated NPC tissue, was obtained from the cell line database (Shanghai FuHeng Biological Technology Co., Ltd.), and were cultured in RPMI-1640 media (Gibco; Thermo Fisher Scientific, Inc.) supplemented with $10 \%$ fetal bovine serum (FBS; Invitrogen; Thermo Fisher Scientific, Inc.), penicillin $(100 \mathrm{U} / \mathrm{ml})$ and streptomycin $(100 \mathrm{U} / \mathrm{ml})$ (both Gibco; Thermo Fisher Scientific, Inc.) in a humidified incubator at $37^{\circ} \mathrm{C}\left(5 \% \mathrm{CO}_{2}\right)$. 
Cell viability assay. C666-1 cells were seeded into a 96-well plate $\left(5 \times 10^{3}\right.$ cells per well) and treated with increasing concentrations of cordycepin $(0,250,500,750$ and $1,000 \mu \mathrm{M}$ for 24-72 h, or cisplatin for $24 \mathrm{~h}$ at $37^{\circ} \mathrm{C}$. Following treatment, cell viability was assessed using the Cell Counting Kit 8 (CCK-8) assay (APExBIO Technology LLC); $10 \mu \mathrm{l} \mathrm{CCK-8} \mathrm{solution} \mathrm{was}$ added, and the cells were incubated for $4 \mathrm{~h}$ at $37^{\circ} \mathrm{C}$. Optical density was detected at $450 \mathrm{~nm}$ using a microplate reader (Thermo Fisher Scientific, Inc.).

Colony formation assay. C666-1 cells were counted, seeded into 12-well plates in triplicate ( 800 cells per well), cultured in RPMI (supplemented with $10 \%$ fetal bovine serum), and treated with cordycepin for up to 14 days as aforementioned. Then, the cells were washed twice with PBS and fixed using methanol for $10 \mathrm{~min}$ at $4^{\circ} \mathrm{C}$. After two additional washes with PBS, the cells were stained with crystal violet for $30 \mathrm{~min}$ at room temperature. The cells were then washed with double-distilled water $\left(\mathrm{ddH}_{2} \mathrm{O}\right)$ to remove the crystal violet, and the colony numbers were counted using ImageJ software (version 1.52; National Institutes of Health), set to the area of colonies above 5 pixel $^{\wedge 2}$ as 1 colony.

Wound-healing assay. C666-1 cells were seeded into 12-well plates and incubated in serum-free medium at $37^{\circ} \mathrm{C}$ for $18 \mathrm{~h}$. The cell monolayers were scratched with a $10-\mu 1$ pipette tip and washed with serum-free RPMI-1640 media (Gibco; Thermo Fisher Scientific, Inc.) to remove cells detached from the plates. The cells were incubated in the presence or absence of cordycepin for 48-72 h (as aforementioned) in medium containing $10 \%$ FBS. Then, the medium was replaced with PBS and images of the cells were captured using a fluorescence inverted microscope (Leica Microsystems, Inc.; magnification, x20) in brightfield mode. The results were quantified using ImageJ software (version 1.52).

Transwell migration assay. To assess cellular migration, $5 \times 10^{4}$ cells were seeded into Transwell inserts in a 24-well plate, with serum-free medium in the upper chambers, and RPMI containing $10 \% \mathrm{FBS}$ added to the lower chambers. The cells were incubated for $24-48 \mathrm{~h}$ at $37^{\circ} \mathrm{C}$, washed once with PBS, and then fixed with $4 \%$ paraformaldehyde for $10 \mathrm{~min}$ at room temperature. The cells were stained with $0.1 \%$ crystal violet for $30 \mathrm{~min}$ at room temperature, and then washed with $\mathrm{dd}_{2} \mathrm{O}$. The non-migrated cells were removed with a cotton swab, and the stained cells were observed by a fluorescence inverted microscope (Leica Microsystems, Inc.; magnification, $\mathrm{x} 20$ ).

RNA extraction, library construction, and sequencing. Total RNA of each cordycepin-treated and control sample was extracted using TRIzol ${ }^{\circledR}$ reagent (Invitrogen; Thermo Fisher Scientific, Inc.), following the manufacturer's instructions. The quality of RNA was assessed on an Agilent 2100 Bioanalyzer (Agilent Technologies, Inc.) and checked using RNase-free agarose gel electrophoresis. Then mRNA was enriched and purified using oligo (dT) beads. The purified mRNA was cut into short fragments using fragmentation buffer (Shanghai Yeasen Biotechnology Co., Ltd.) and reverse transcribed into cDNA using random primers. Second-strand cDNA were synthesized using DNA polymerase I, RNase H, dNTP and buffer. Then the cDNA fragments were purified using the QIAquick PCR extracting kit (Qiagen). After performing end repair and adding poly (A), the cDNA fragments were ligated using Illumina sequencing adapters. The ligation products were enriched via PCR amplification to construct the cDNA library template (NovaSeq 6000 S4 Reagent Kit v1.5; 300 cycles; cat. no. 20028312). Finally, the library (10 pM per sample) was sequenced by $150 \mathrm{bp}$ paired end sequencing using the Illumina Novaseq 6000 (Illumina Inc.) by Guangzhou Gene Denovo Biotechnology Co. Ltd.

Transcriptome mapping, annotation and differential expression analyses. Sequencing reads were edited for quality and cleaned using fastp (version 0.18.0, https://github. com/OpenGene/fastp). Clean data were mapped to the Homo sapiens (human) genome (GRCh38.p13) using HISAT2 (version 2.4, http://daehwankimlab.github.io/hisat2/). The mapped reads of each sample were assembled using StringTie (version 1.3.1, http://ccb.jhu.edu/software/stringtie) using a reference-based approach. For each transcription region, a FPKM (fragment per kilobase of transcript per million mapped reads) value was calculated to quantify its expression abundance and variation using RSEM software (http://deweylab.biostat.wisc.edu/rsem). Differentially expressed genes were identified using DESeq2 (http://www. bioconductor.org/) and edgeR (http://www.rproject.org/) package, with a threshold false discovery rate $<0.05$, and absolute value of the $\log _{2}$ fold change $\geq 1$. All expressed genes were functionally annotated against the NCBI non-redundant protein database using the BLAST algorithm with a cut-off E-value $\leq 10^{-5}$. The genes were also subjected to classification and enrichment analyses of Gene Ontology (GO) functions and Kyoto Encyclopedia of Genes and Genomes (KEGG) pathways. GO and KEGG classification were performed using Gene Ontology database (http://geneontology.org/) and the KEGG automatic annotation server (https://www. genome.jp/kegg/), respectively.

Western blotting. Cells were harvested and lysed in RIPA buffer (Sangon Biotech Co., Ltd.) supplemented with protease inhibitor ( $1 \%$ phenylmethylsulfonyl fluoride) at $4^{\circ} \mathrm{C}$, for $30 \mathrm{~min}$, and then centrifuged at $10,309 \mathrm{x} \mathrm{g}$ for $15 \mathrm{~min} 4^{\circ} \mathrm{C}$. The protein concentrations of the lysates were measured on a spectrophotometer using a BCA Protein Assay Kit (Sangon Biotech Co., Ltd.). The remainder were added to a $5 \mathrm{X}$ loading buffer at 1:4 (Sangon Biotech Co., Ltd.) and heated at $95^{\circ} \mathrm{C}$ for $5 \mathrm{~min}$. Next, $50 \mu \mathrm{g}$ protein per lane was electrophoretically separated by $10 \%$ SDS-PAGE, and transferred to PVDF membranes. The membranes were blocked using 5\% non-fat milk for $1 \mathrm{~h}$ at room temperature, and the incubated with primary antibodies against GAPDH $(1: 2,000$; cat. no. AP0063; Bioworld Technology, Inc.) ERK1/2 (1:1,000; cat. no.137F5; Cell Signaling Technologies, Inc.), p-ERK1/2 (1:1,000; cat. no.9101; Cell Signaling Technologies, Inc.) and $\beta$-catenin (1:1,000; cat. no. ab16051; Abcam) in 1X TBS with $0.05 \%$ Tween (TBS-T), at $4^{\circ} \mathrm{C}$ overnight. The membranes were then incubated with HRP-conjugated secondary antibodies (anti-rabbit; 1:5,000; cat. no. 7074; Cell Signaling Technologies, Inc.) in $1 \mathrm{X}$ TBS-T at room temperature for $1 \mathrm{~h}$. The proteins 
Table I. Primers for qPCR.

\begin{tabular}{ll}
\hline Name & \multicolumn{1}{c}{ Sequence (5'-3') } \\
\hline SELE & \\
Forward & TCAAGGGCAGTGGACACAGCAA \\
Reverse & GGAAACTGCCAGAAGCACTAGG \\
MUC20 & \\
Forward & AGAGTGGCAGAAAGGCTGATGC \\
Reverse & CTGATGTCCGTTAGCCTCTCCT \\
ACTL10 & \\
Forward & GCCAGTTTCAGCGTGGGTAACG \\
Reverse & CAGCGTTTTGGGCATCTTCTGC \\
EFHD1 & \\
Forward & GAGGGTGTCAAAGGTGCCAAGA \\
Reverse & TGAGTTTCTGGAAGGCTGCCTG \\
RGS9 & \\
Forward & CAACGATGCCATCATGTCAGGC \\
Reverse & CCATCGTTCCACTCGCATCTTG \\
GAPDH & \\
Forward & AACATCATCCCTGCCTCTACTG \\
Reverse & CCTCCGACGCCTGCTTCAC \\
\hline
\end{tabular}

SELE, selectin E; MUC20, mucin 20, cell surface associated; ACTL10, actin like 10; RGS9, regulator of G protein signaling 9; EFHD1, EF-hand domain family member D1.

were visualized using a chemiluminescence (ECL) reagent (Pierce ECL Western Blotting Substrate; Thermo Fisher Scientific, Inc.).

Reverse transcription-quantitative $(R T-q) P C R$. RNA sequencing (RNA-seq) results were validated using RT-qPCR. Total RNA was extracted using TRIzol ${ }^{\circledR}$ reagent (Invitrogen; Thermo Fisher Scientific, Inc.) per the manufacturer's instructions. Reverse transcription and qPCR were performed using an RT Kit (Hunan Accurate Bio-Medical Co., Ltd.) and TB Green PCR Master Mix (Takara Bio, Inc.), respectively, according to the manufacturers' protocols. The qPCR reaction was carried out using a three-step method on a LightCycler 480 Instrument II (Roche Diagnostics): Initial denaturation at $95^{\circ} \mathrm{C}$ for $5 \mathrm{~min}$, then amplification at $95^{\circ} \mathrm{C}$ for $5 \mathrm{sec}, 58^{\circ} \mathrm{C}$ for $30 \mathrm{sec}$ and $72^{\circ} \mathrm{C}$ for $20 \mathrm{sec}$ (a total of 40 cycles). The oligonucleotide primers are displayed in Table I; GAPDH was used as the housekeeping gene to normalize the expression levels of mRNA, which were quantified using the $2^{-\Delta \Delta \mathrm{Cq}}$ method (18).

Statistical analysis. Each experiment was repeated three times independently, and the data are expressed as the mean \pm standard deviation. The data were analyzed using GraphPad Prism 6.02 (GraphPad Software, Inc.); differences between two groups were analyzed using the unpaired Student's t-test, and differences among $\geq 3$ groups were compared using one-way ANOVA followed by Dunnett's multiple comparisons test. $\mathrm{P}<0.05$ was considered to indicate a statistically significant difference.

\section{Results}

Cordycepin inhibits the proliferation of NPC cells. C666-1 cells, EBV-positive NPC cells taken from undifferentiated NPC tissue (19), were used to investigate the effects of cordycepin on NPC cell proliferation. Increasing concentrations of cordycepin $(250,500$ and $1,000 \mu \mathrm{M})$ were added to the culture media for 24, 48 and $72 \mathrm{~h}$, and C666-1 cell viability was determined using a CCK- 8 assay. The results demonstrated that cordycepin decreased the viability of C666-1 cells in a dose-dependent manner, with an $\mathrm{IC}_{50}$ of $1.37 \mathrm{mM}$ at $24 \mathrm{~h}$, $842.5 \mu \mathrm{M}$ at $48 \mathrm{~h}$ and $546.9 \mu \mathrm{M}$ at $72 \mathrm{~h}$ (Fig. 1A). Furthermore, a colony formation assay revealed that $500 \mu \mathrm{M}$ cordycepin significantly inhibited colony formation, and that $1 \mathrm{mM}$ cordycepin completely inhibited clone formation (Fig. 1B and C). These results indicate that cordycepin inhibited the proliferation of NPC cells.

Cordycepin inhibits the migration of NPC cells. To investigate the effects of cordycepin on the migration of C666-1 cells, wound-healing and Transwell assays were performed. Cells treated with 250 and $500 \mu \mathrm{M}$ cordycepin showed inhibited wound-healing ability (Fig. 2A and B). Furthermore, $500 \mu \mathrm{M}$ cordycepin significantly inhibited C666-1 cell migration through the Transwell insert membrane (Fig. 2C). These results indicate that cordycepin inhibited the migration of NPC cells.

Cordycepin enhances the effects of cisplatin on NPC cells. Next, the potential for cordycepin to enhance the chemotherapeutic effects of cisplatin was investigated in NPC cells. An additional colony formation assay was performed, and a relatively low concentration of cisplatin that did not affect cell viability $(0.5 \mu \mathrm{g} / \mathrm{ml})$ was used. The assay revealed that the colony formation ability of NPC cells was completely inhibited following combined treatment with cordycepin and cisplatin (Fig. 3). This result indicates that cordycepin enhanced the inhibitory effects of cisplatin on NPC cells.

Changes in the global gene expression profile of C666-1 cells after cordycepin treatment. To investigate how cordycepin regulates downstream signaling pathways, transcriptome RNA-seq analyses were performed to compare cells treated with $500 \mu \mathrm{M}$ cordycepin for $48 \mathrm{~h}$ with untreated control cells (Fig. 4A). In total, 20,295 genes were identified, and the expression levels of 1,541 genes were altered to varying degrees. Among the differentially expressed genes, 72 were significantly different $\left(\log _{2} \mid\right.$ FoldChange $\left.\left.1>2\right), P<0.05\right)$, including 35 downregulated and 37 upregulated genes (Fig. 4B). The top upregulated and downregulated genes are presented in Fig. 4C. This experiment showed global gene expression alterations following cordycepin treatment.

Functional annotation of differentially expressed genes after cordycepin treatment. To map changes in the downstream signaling pathways, GO and KEGG pathway enrichment analyses were performed on all significantly differentially expressed genes (above a 1.5-fold change). An overview of the top functions is provided in Fig. 5. Both up- and downregulated genes were enriched, revealing a global map of signaling transduction changes in the cells. 
A

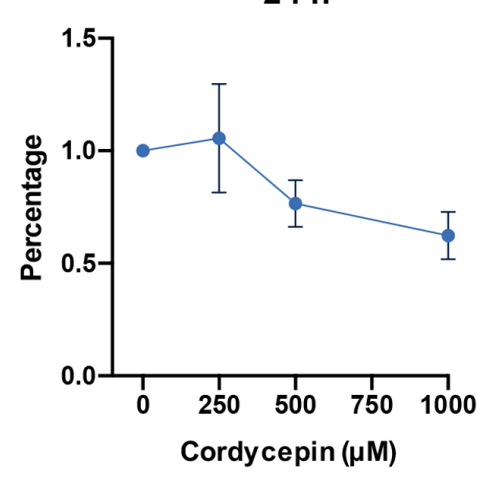

$48 \mathrm{~h}$

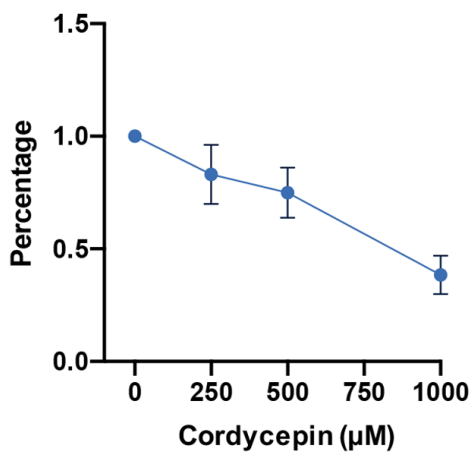

$72 \mathrm{~h}$

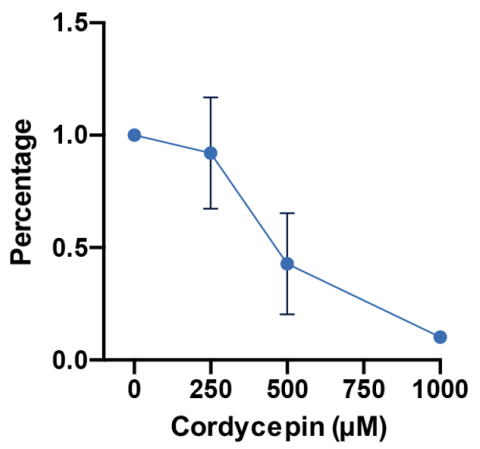

B

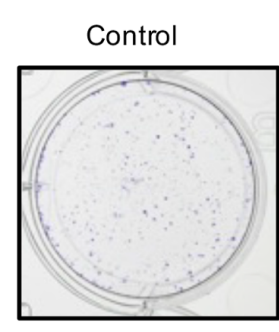

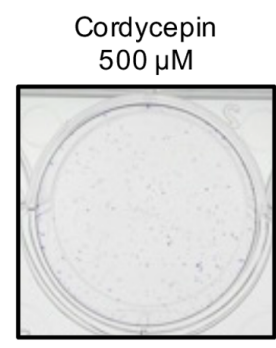

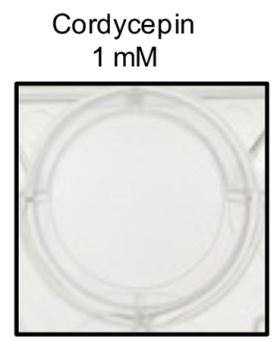

C

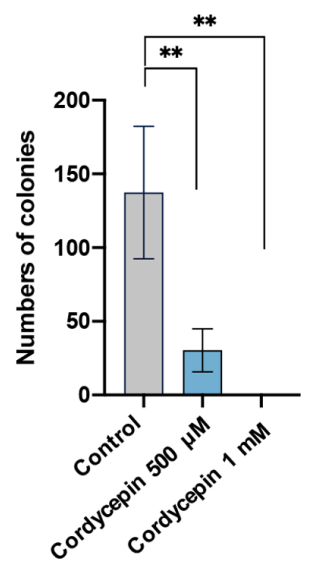

Figure 1. Cordycepin inhibits the proliferation of C666-1 cells. (A) C666-1 cells were cultured in the presence of 250,500 and 1,000 $\mu \mathrm{M}$ cordycepin for up to $72 \mathrm{~h}$, and cell viability was measured at different time points $(24,48$ and $72 \mathrm{~h})$ using the Cell Counting Kit 8 assay. Cell viability decreased in a dose-dependent manner after cordycepin treatment. (B) C666-1 cells were treated with $500 \mu \mathrm{M}$ and $1 \mathrm{mM}$ cordycepin for 2 weeks, and colonies were stained and counted. (C) Colony numbers were counted and compared with the control group. Each experiment was performed three times and the data are expressed as the mean $\pm \mathrm{SD} .{ }^{* *} \mathrm{P}<0.01$.

A detailed enrichment figure reveals KEGG pathways that could affect the proliferation and migration of cancer cells, including 'cell adhesion molecules', 'PD-L1 expression and PD-1 checkpoint pathway in cancer', 'T cell receptor signaling pathway', 'TNF signaling pathway', and 'VEGF signaling pathway'. GO term enrichment results highlighted biological processes related to cell proliferation and migration, such as "regulation of the JNK cascade', 'positive regulation of the stress-activated MAPK cascade' and 'cell adhesion' (Fig. 6A).

Next, Gene Set Enrichment Analysis of the KEGG pathways was performed to identify those that were the most inhibited by cordycepin treatment. These included 'ribosome', 'oxidative phosphorylation', 'protein export', 'PPAR signaling pathway', 'glutathione metabolism' and 'central carbon metabolism in cancer' (Fig. 6B).

These analyses indicate downstream signaling pathway alterations that are regulated by cordycepin.

Cordycepin may inhibit NPC cells through the ERK and $\beta$-catenin signaling pathways. To verify the RNA-seq data, four significantly differentially expressed genes identified from RNA-seq were selected and verified via RT-qPCR. Selectin E and actin like 10 were upregulated by $500 \mu \mathrm{M}$ cordycepin treatment, while regulator of $\mathrm{G}$ protein signaling 9 
A
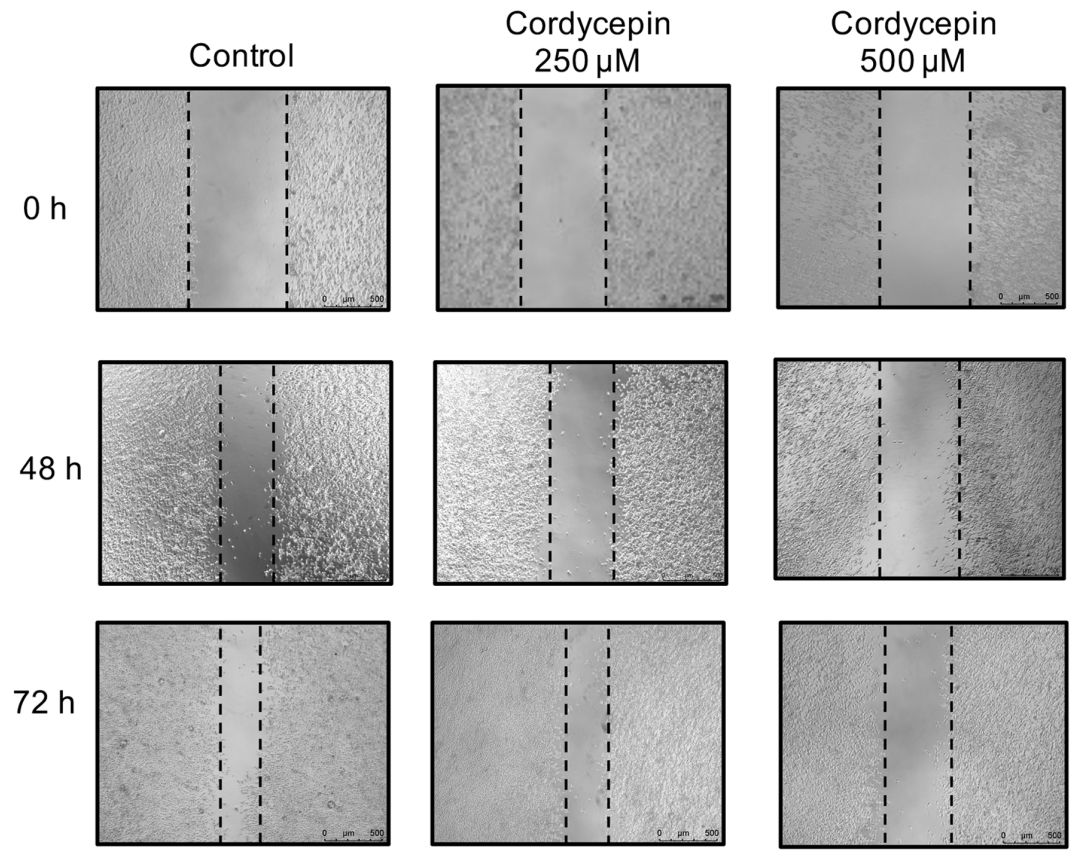

B
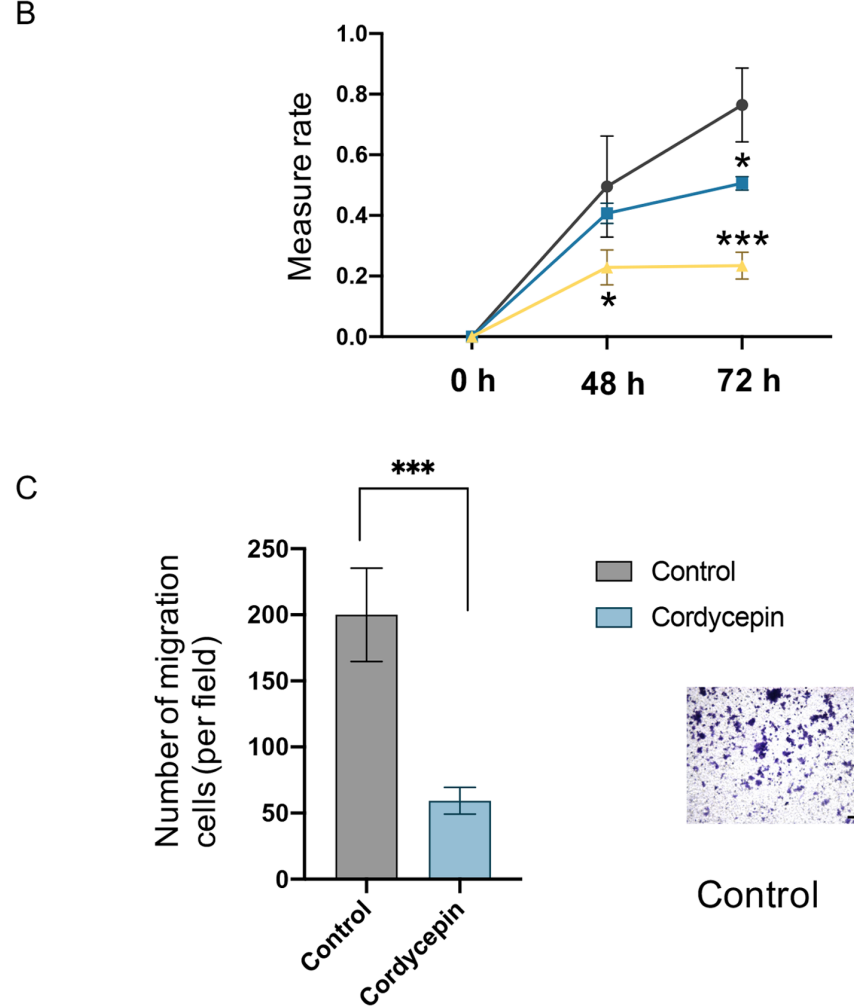

Control

Cordycepin

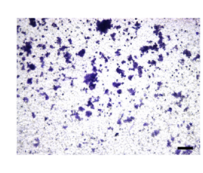

Control
- Control
$-\quad$ Cordycepin $250 \mu \mathrm{M}$
$-\quad$ Cordycepin $500 \mu \mathrm{M}$

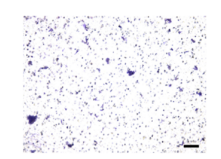

Cordycepin $500 \mu \mathrm{M}$

Figure 2. Cordycepin inhibits the migration of C666-1 cells. (A) Wound-healing ability of C666-1 cells was measured after treatment with 250 and 500 $\mu \mathrm{M}$ cordycepin for up to $72 \mathrm{~h}$. Images were captured at the indicated time points after initial wound generation. (B) Migration rate was calculated, and $500 \mu \mathrm{M}$ cordycepin significantly inhibited the migration of C666-1 cells relative to the untreated control. (C) The migration ability of C666-1 cells was measured using a Transwell migration assay. C666-1 cells were treated with $500 \mu \mathrm{M}$ cordycepin and those that migrated through the polycarbonate membrane were stained and counted. Scale bar, $100 \mu \mathrm{m}$. Each experiment was performed three times and the data are expressed as the mean $\pm \mathrm{SD}$. ${ }^{*} \mathrm{P}<0.05$ and ${ }^{* * *} \mathrm{P}<0.001$.

and EF-hand domain family member D1 were downregulated, which was consistent with the sequencing results (Fig. 7A). To investigate whether cordycepin affects the MAPK/ERK and Wnt signaling pathways, western blotting was performed to evaluate the protein expression levels of ERK1/2 and phosphorylated-ERK1/2 (p-ERK) at different concentrations of cordycepin for $48 \mathrm{~h}$. The results showed a similar expression level of p-ERK at $500 \mu \mathrm{M}$ cordycepin compared with the control group, while significant inhibition of ERK and p-ERK1/2 was found after treatment with $750 \mu \mathrm{M}$ cordycepin, which is close to the $\mathrm{IC}_{50}$ value at this time point (Fig. 7B). The protein expression of $\beta$-catenin was also arrested (relative to the untreated control group) following cordycepin treatment. Different expression levels of $\beta$-catenin were detected at low 
A

Control

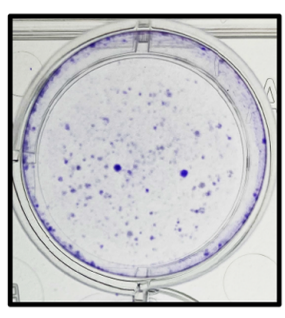

Cisplatin $0.5 \mu \mathrm{g} / \mathrm{ml}$

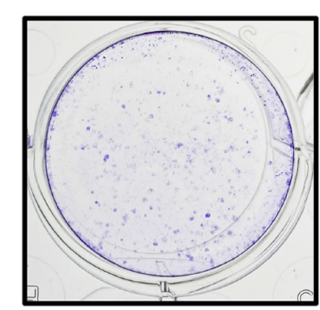

Cisplatin

$0.5 \mu \mathrm{g} / \mathrm{ml}$

$+$

Cordycepin

$500 \mu \mathrm{M}$

B

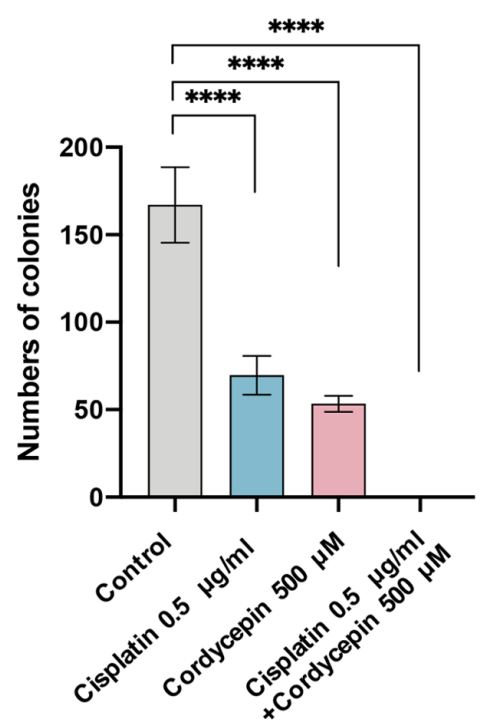

Figure 3. Cordycepin and cisplatin combination treatment inhibits the colony formation ability of C666-1 cells. (A) C666-1 cells were treated with $0.5 \mu \mathrm{g} / \mathrm{ml}$ cisplatin, $500 \mu \mathrm{M}$ cordycepin, or both for 2 weeks, and colonies were counted and compared with the untreated cells. (B) Low concentrations of cordycepin and cisplatin inhibited the colony formation of C666-1 cells, while the combination of the two drugs completely inhibited colony formation. Each experiment was performed three times and the data are expressed as the mean $\pm \mathrm{SD}$. ${ }^{* * * *} \mathrm{P}<0.0001$.

$(250 \mu \mathrm{M})$ and high (500 and $750 \mu \mathrm{M})$ concentrations, which suggests different regulation mechanisms of signaling transduction. These results indicate that cordycepin may inhibit proliferation and migration of NPC cells through the ERK and $\beta$-catenin signaling pathways.

\section{Discussion}

Natural products such as gingerol, curcumin and gambogic have been widely studied, and are considered candidates for treating different types of cancer $(9,10)$. Cordycepin is the primary compound extracted from Cordyceps fungi, which has been used as a dietary supplement in some Asian countries for hundreds of years (11). Furthermore, the anticancer properties of cordycepin have been identified in various types of malignancy, including breast, liver and lung cancer $(13,15,17,20,21)$.

Cordycepin is an adenosine derivative that can regulate cell functions through adenosine receptors, death receptors or epidermal growth factor receptor (EGFR) (21-23). The extract inhibits the proliferation, migration, invasiveness and cell cycle of non-small-cell lung cancer cells $(15,24)$. In addition, drug-resistant lung cancer cell lines with EGFR mutations are more sensitive to cordycepin treatment than those without EGFR mutations, and this effect may be produced through an interaction with AMPK, whereby cordycepin activates the signaling pathway downstream of AMPK to induce apoptosis (15). Cordycepin also inhibits pancreatic cancer cell proliferation by targeting fibroblast growth factor receptor 2 to block the MAPK signaling pathway (25).

In the present study, the effects of cordycepin on NPC cells were investigated, where it was found to inhibit the proliferation of EBV-positive NPC cells, and to augment the killing effects of low concentrations of cisplatin. Previous studies have indicated that cordycepin enhances the apoptotic effects of cisplatin in head and neck tumor cell lines, and that co-treatment with the two drugs significantly increases the cleavage of caspase 38 and 9, and PARP, and activates the 
A

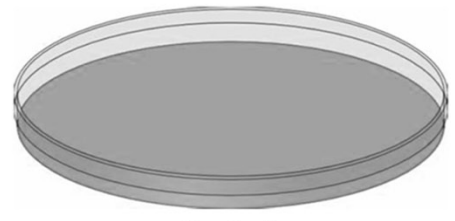

Control

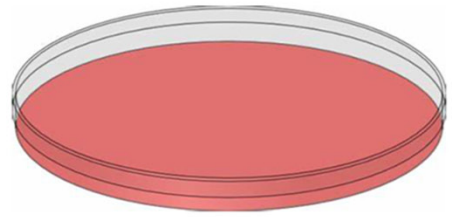

Cordycepin $500 \mu \mathrm{M}$

Total RNA extraction \& RNA sequencing

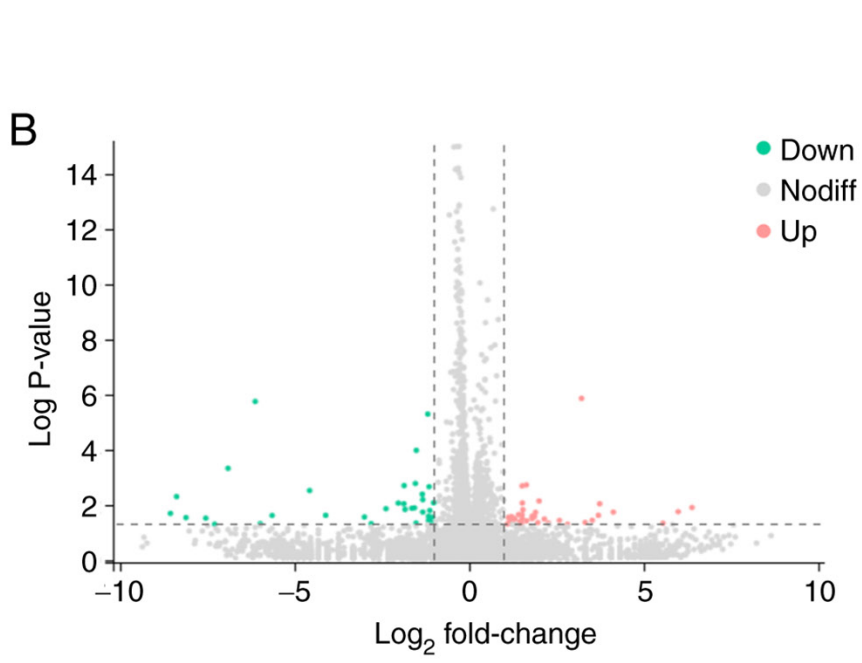

C

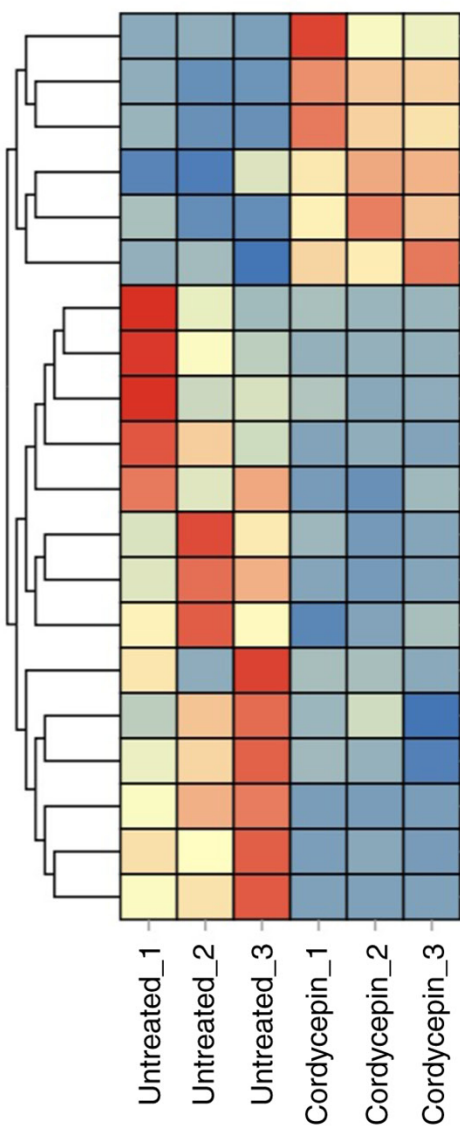

SELE

MUC20

ACTL10

GRIN2C

BIVM-ERCC5

SPDYE2

SPARCL1

AC242842.3

TUBA1A

C16orf46

RGS9

EFHD1

AL441992.2

AL139300.1

SPDYE2B

LY75-CD302

AC010323.1

AC0050202.2

GABRP

AL033529.1
35

1469

Figure 4. RNA-seq of the transcriptome profiles of C666-1 cells after treatment with cordycepin. (A) C666-1 cells were treated (or not) with 500 $\mu$ M cordycepin, total RNA was extracted and RNA-seq was performed; three biological repeats were performed and sequenced. (B) Volcano plot and Venn diagram indicating several 37 significantly upregulated and 35 significantly downregulated genes $(\mathrm{P}<0.05 ; \mathrm{Log} 2 \mathrm{FCl}>2)$ in the cordycepin-treated group compared with the control group. (C) Heatmap of the most differentially expressed genes. RNA-seq, RNA sequencing.

MAPK pathway (26). Cordycepin also enhances the chemotherapeutic effects of cisplatin against esophageal cancer. Co-treatment inhibited cellular proliferation, migration and metastatic capacity, and induced the apoptosis of esophageal cancer cells by repressing the expression of $\mathrm{p}-\mathrm{PI} 3 \mathrm{~K}, \mathrm{p}-\mathrm{AKT}$, caspase 3 and $\mathrm{Bcl} 2$, while activating p-AMPK, cleaved caspase 3 and $\operatorname{Bax}(27)$. In the current study, the underlying mechanisms of cordycepin treatment in NPC cells were investigated via RNA-seq analysis. GO and KEGG enrichment analyses highlighted proliferation- and migration-related pathways, including 'cell adhesion molecules', 'VEGF signaling pathway', and 'regulation of the JNK cascade', which also suggests that cordycepin may affect NPC cell function through these downstream pathways. qPCR analysis validated four of the most significantly differentially expressed genes identified from RNA-seq, which supports the reliability of the results.

Western blot analyses revealed that at $500 \mu \mathrm{M}$ cordycepin, the levels of $\mathrm{p}$-ERK1/2 increased, while at $750 \mu \mathrm{M}$ cordycepin, the levels of total ERK1/2 and p-ERK1/2 were reduced. This suggests that $750 \mu \mathrm{M}$ cordycepin inhibits proliferation by arresting the ERK pathway. A previous study suggested that cordycepin induced the apoptosis of head and neck squamous cell carcinoma cells through the phosphorylation of ERK proteins (26), which could explain the early increase in p-ERK1/2; the present study observations for NPC cells at $500 \mu \mathrm{M}$ cordycepin are consistent with these findings. 
A

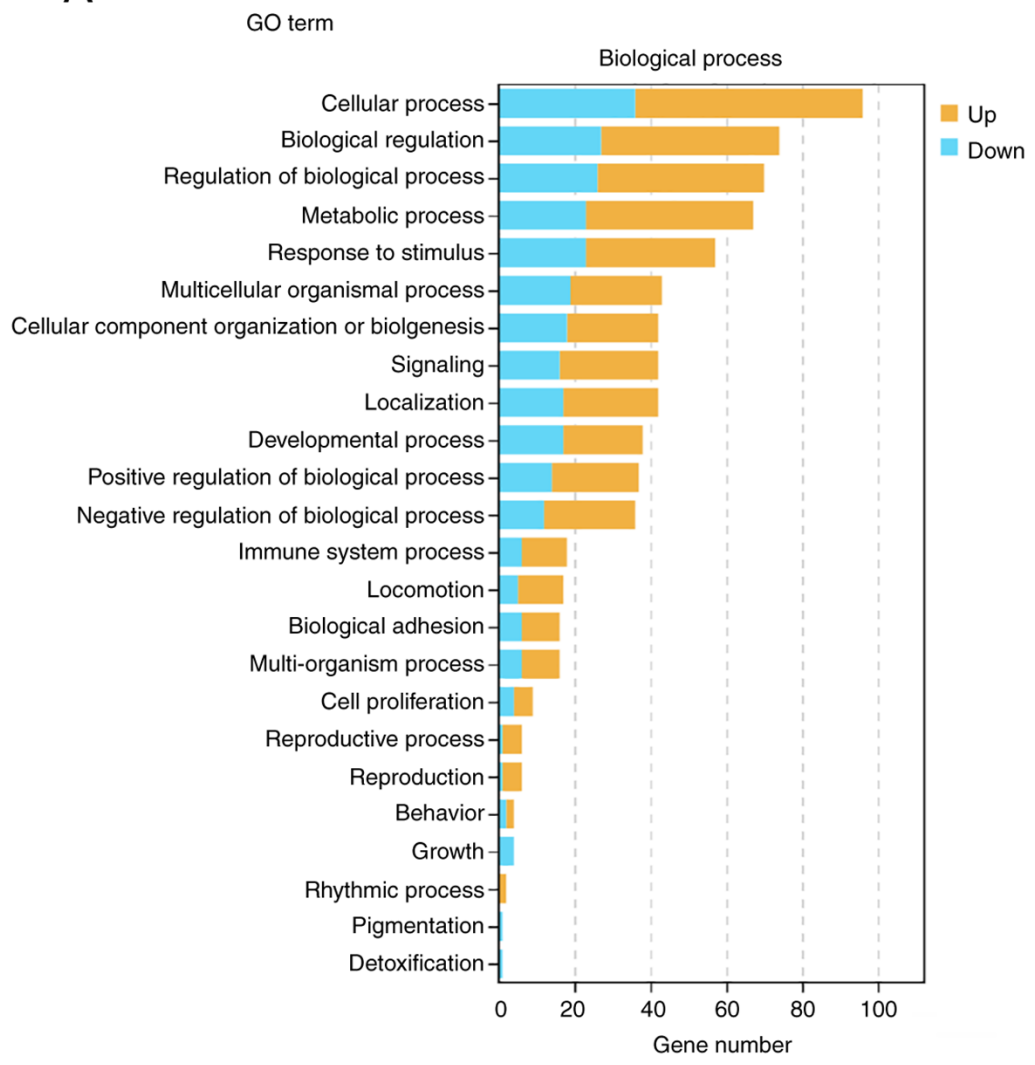

$\mathrm{B}$

KEGG pathway
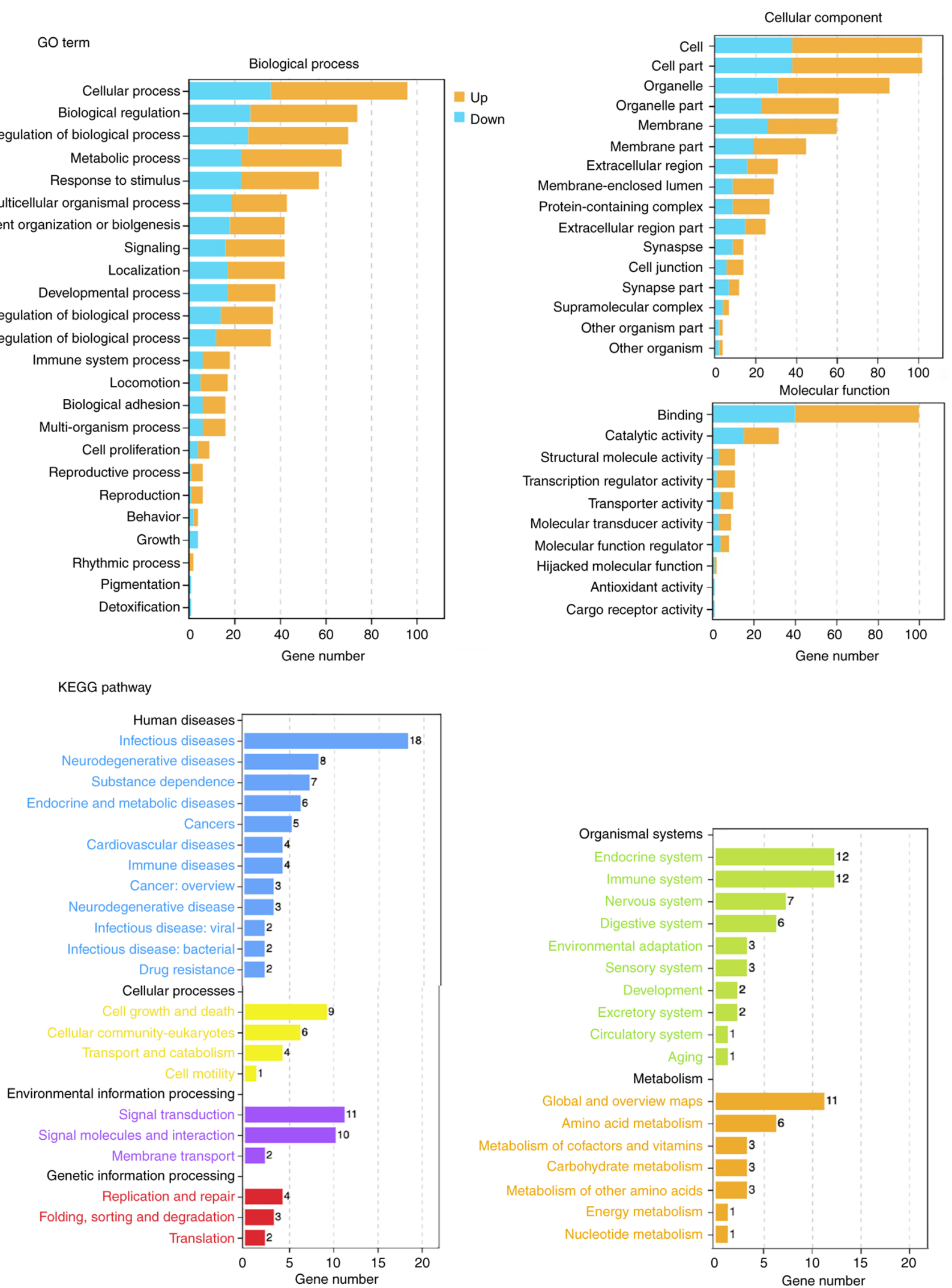

Figure 5. Enrichment analysis of C666-1 cells after treatment with cordycepin. (A) Top enrichment GO terms of biological process, cellular component and molecular function. (B) Top KEGG pathway categories, including 'human diseases', 'cellular process', 'environmental information processing', 'genetic information processing', 'organismal systems' and 'metabolism'. GO, gene ontology; KEGG, Kyoto Encyclopedia of Genes and Genomes.

Cordycepin may retard migration by inhibiting the expression of $\beta$-catenin, a key constituent of the Wnt signaling pathway and a promising drug target for various cancer types $(28,29)$.
Finally, the results of the current study revealed that when sufficient cordycepin is applied, it inhibits the expression of ERK1/2 and $\beta$-catenin, which represses the downstream 
A

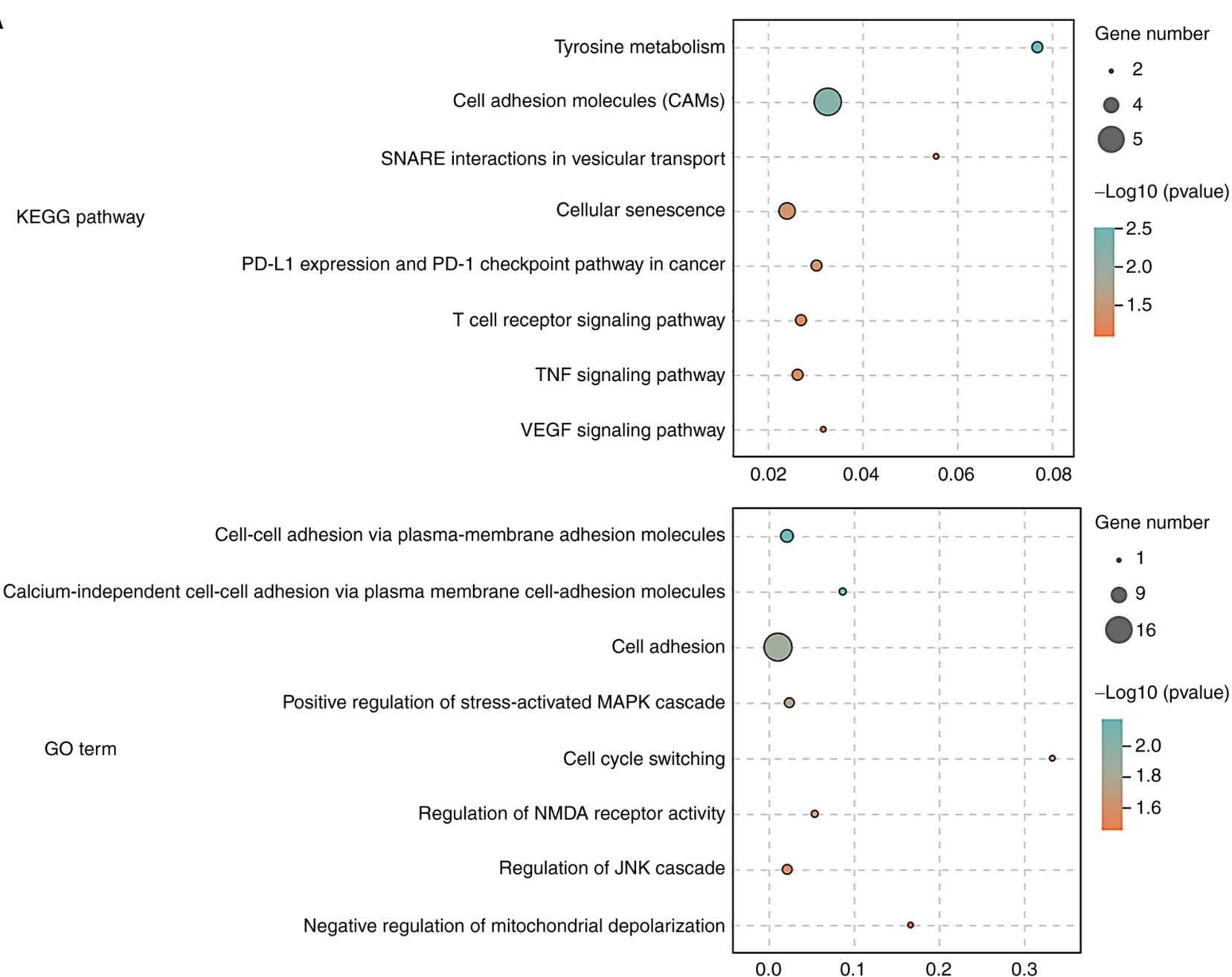

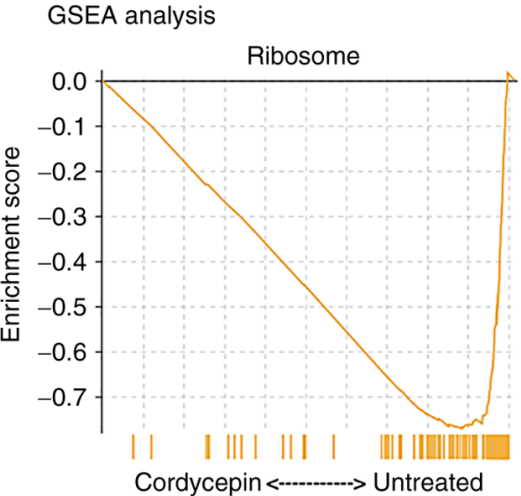

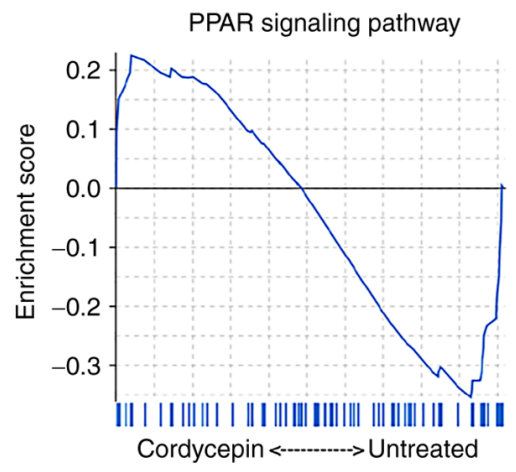

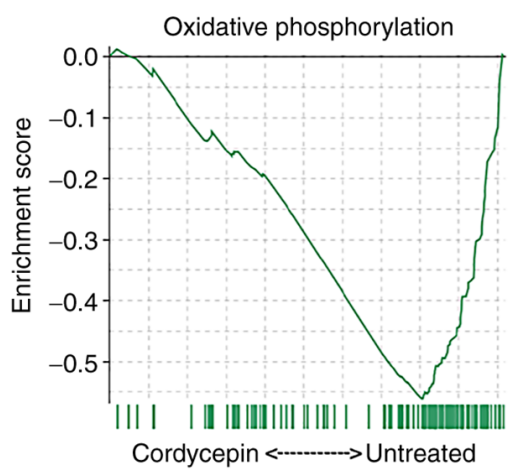

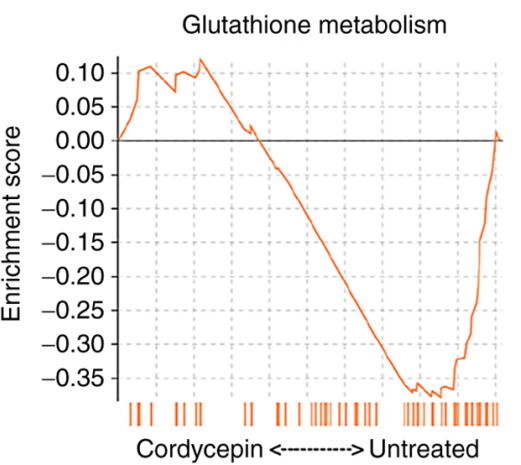

Protein export

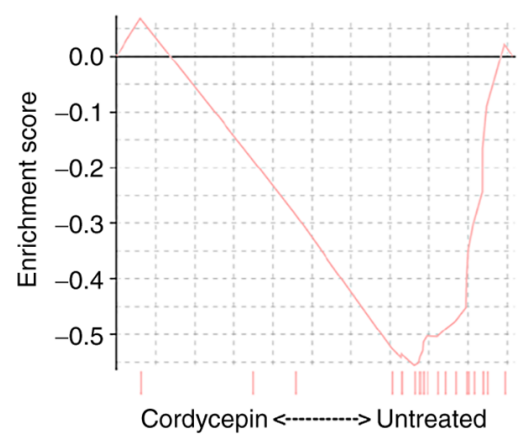

Central carbon metabolism in cancer

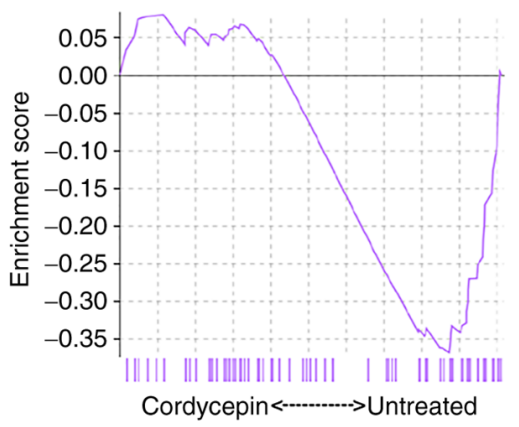

Figure 6. GO, KEGG, and GSEA enrichment analyses revealed changes in downstream signaling of C666-1 cells after cordycepin treatment. (A) KEGG pathway and GO term enrichment analyses indicating significantly enriched pathways and biological processes in up- and downregulated genes after cordycepin treatment; $\mathrm{P}<0.05$. (B) GSEA analyses revealing the pathways downregulated by cordycepin treatment. GO, gene ontology; KEGG, Kyoto Encyclopedia of Genes and Genomes; GSEA, Gene Set Enrichment Analysis. 
A

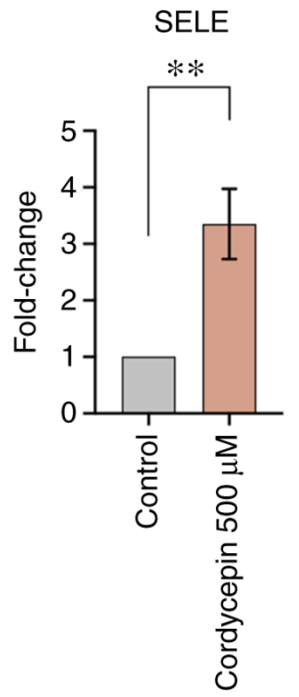

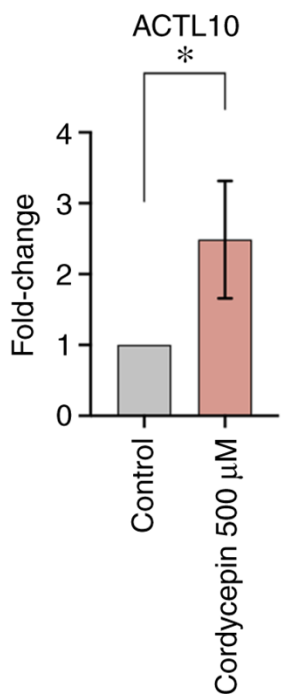

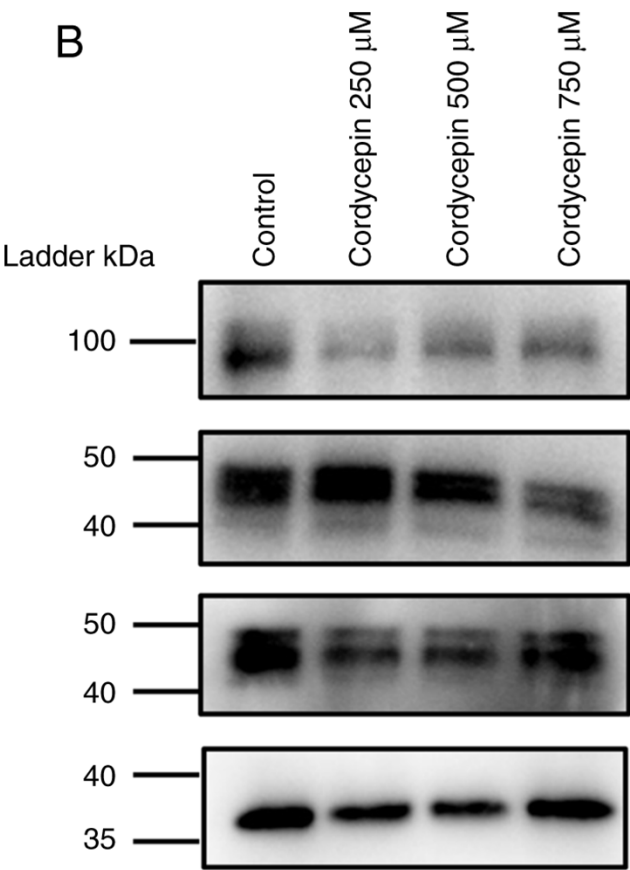

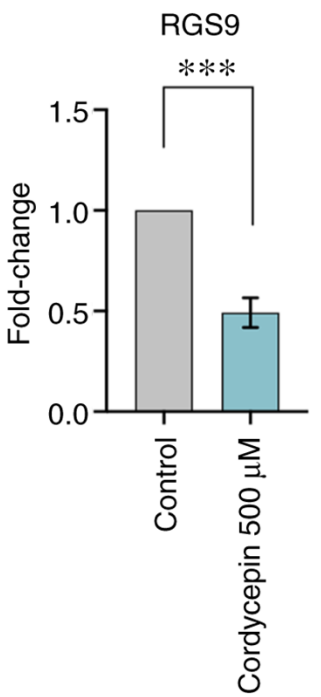

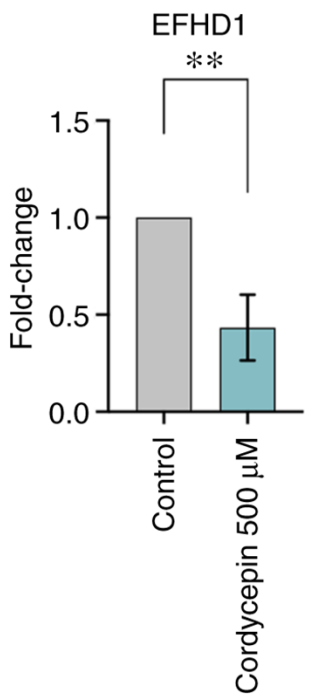

C

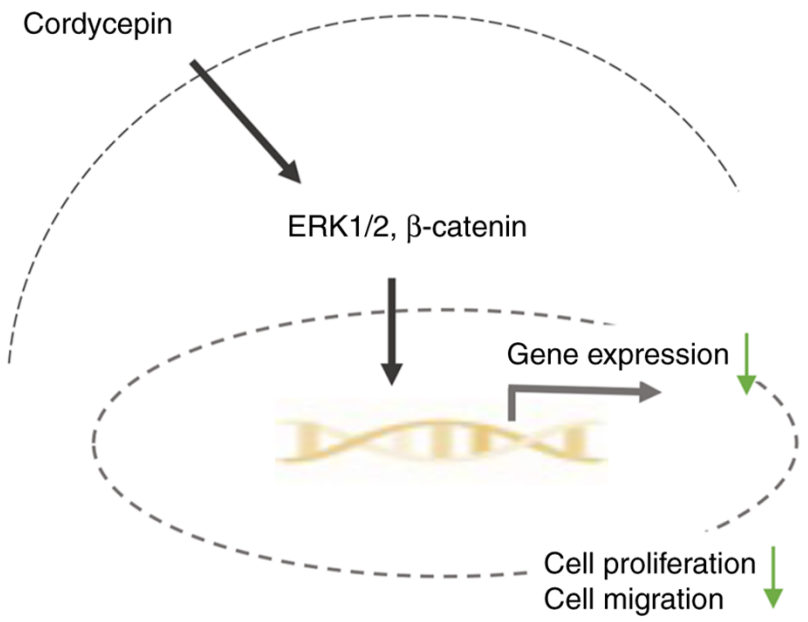

Figure 7. Changes in downstream signaling pathways in C666-1 cells after cordycepin treatment. (A) Reverse trasnscription-quantitative PCR revealed that SELE and ACTL10 were upregulated by cordycepin, while RGS9 and EFHD were downregulated. (B) Western blotting revealed that the protein levels of ERK1/2 and p-ERK1/2 were reduced in cells treated with $750 \mu \mathrm{M}$ cordycepin. (C) Diagram showing a possible cordycepin-induced signaling pathway. ${ }^{*} \mathrm{P}<0.05,{ }^{* *} \mathrm{P}<0.01$ and ${ }^{* * * *} \mathrm{P}<0.001$. p-, phosphorylated. SELE, selectin E; ACTL10, actin like 10; RGS9, regulator of G protein signaling 9; EFHD1, EF-hand domain family member D1.

signaling pathway to reduce the proliferation and migration of NPC cells (Fig. 7C).

Although natural products, such as cordycepin, show promising anticancer potential, the majority of studies have only considered in vitro systems. A few studies have investigated the effects of cordycepin in cancer-bearing mice. In a human oral squamous cell carcinoma xenograft model, cordycepin (via intraperitoneally injection) inhibited tumor growth without affecting weight, or the function of the liver or kidney (30). Another study that used a xenograft model of cholangiocarcinoma demonstrated the anticancer ability of cordycepin in vivo (31). However, further studies investigating the efficacy of cordycepin for treating NPC in vivo are required.

The present study only explored the effects of cordycepin in an NPC cell line, but not in normal control cells. Future studies will investigate how cordycepin regulates downstream molecules in EBV-positive and -negative NPC cells, and will compare them to normal nasopharynx epithelial cell lines via transcriptome and proteome experiments.

In conclusion, the present study demonstrated the anticancer effects of cordycepin in EBV-positive NPC cells. The combination of cordycepin and cisplatin may allow NPC treatment that goes beyond single cisplatin chemotherapy. Moreover, the inhibitory effects of cordycepin in NPC cells resulted from the activation of the MAPK/ERK and $\beta$-catenin signaling pathways.

\section{Acknowledgements}

Not applicable. 


\section{Funding}

The present study was supported by grants from the National Natural Science Foundation of China (grant no. 82002885 and 52007001), the China Postdoctoral Science Foundation (grant no. 2021M692159), and the Sanming Project of Medicine in Shenzhen (grant no. SZSM201612076).

\section{Availability of data and materials}

The datasets used and/or analyzed during the current study are available from the corresponding author on reasonable request.

\section{Authors' contributions}

YZ, YL, XS and $\mathrm{HH}$ were responsible for study design and conceptualization. YZ performed most of the experiments. $\mathrm{YZ}$ and $\mathrm{HH}$ confirm the authenticity of all the raw data. $\mathrm{YZ}$, $\mathrm{XM}$ and WY were responsible for data analysis. YZ wrote the manuscript. XS and $\mathrm{HH}$ reviewed and edited the manuscript. All authors read and approved the final manuscript.

\section{Ethics approval and consent to participate}

Not applicable.

\section{Patient consent for publication}

Not applicable.

\section{Competing interests}

The authors declare that they have no competing interests.

\section{References}

1. Chen YP, Chan ATC, Le QT, Blanchard P, Sun Y and Ma J: Nasopharyngeal carcinoma. Lancet 394: 64-80, 2019.

2. Bray F, Ferlay J, Soerjomataram I, Siegel RL, Torre LA and Jemal A: Global cancer statistics 2018: GLOBOCAN estimates of incidence and mortality worldwide for 36 cancers in 185 countries. CA Cancer J Clin 68: 394-424, 2018.

3. Lee AW, Ng WT, Chan LL, Hung WM, Chan CC, Sze HC, Chan OS, Chang AT and Yeung RM: Evolution of treatment for nasopharyngeal cancer - success and setback in the intensity-modulated radiotherapy era. Radiother Oncol 110: 377-384, 2014.

4. Colevas AD, Yom SS, Pfister DG, Spencer S, Adelstein D, Adkins D, Brizel DM, Burtness B, Busse PM, Caudell JJ, et al: NCCN Guidelines Insights: Head and Neck Cancers, Version 1.2018. J Natl Compr Canc Netw 16: 479-490, 2018.

5. Zhang Y, Chen L, Hu GQ, Zhang N, Zhu XD, Yang KY, Jin F, Shi M, Chen YP, Hu WH, et al: Gemcitabine and Cisplatin Induction Chemotherapy in Nasopharyngeal Carcinoma. N Engl J Med 381: 1124-1135, 2019.

6. Huang YM, Qiao SQ, Lu L, Chen WP, Li SL and Qi CH: Gemcitabine combined with cisplatin vs. taxane, cisplatin, and fluorouracil in the treatment of locally advanced nasopharyngea carcinoma: A retrospective case-control study. Eur Rev Med Pharmacol Sci 24: 7655-7663, 2020.

7. Lu Y, Chen D, Liang J, Gao J, Luo Z, Wang R, Liu W, Huang C, Ning X, Liu M, et al: Administration of nimotuzumab combined with cisplatin plus 5-fluorouracil as induction therapy improves treatment response and tolerance in patients with locally advanced nasopharyngeal carcinoma receiving concurrent radiochemotherapy: A multicenter randomized controlled study. BMC Cancer 19: 1262, 2019.
8. Jin T, Qin WF, Jiang F, Jin QF, Wei QC, Jia YS, Sun XN Li WF and Chen XZ: Cisplatin and Fluorouracil Induction Chemotherapy With or Without Docetaxel in Locoregionally Advanced Nasopharyngeal Carcinoma. Transl Oncol 12: 633-639, 2019.

9. Li Y, Li S, Meng X, Gan RY, Zhang JJ and Li HB: Dietary Natural Products for Prevention and Treatment of Breast Cancer. Nutrients 9: E728, 2017.

10. Luo H, Vong CT, Chen H, Gao Y, Lyu P, Qiu L, Zhao M, Liu Q, Cheng Z, Zou J, et al: Naturally occurring anti-cancer compounds: Shining from Chinese herbal medicine. Chin Med 14: 48, 2019.

11. Tuli HS, Sharma AK, Sandhu SS and Kashyap D: Cordycepin: A bioactive metabolite with therapeutic potential. Life Sci 93: 863-869, 2013.

12. Zhu X, Shen H, Yin X, Long L, Xie C, Liu Y, Hui L, Lin X, Fang Y, Cao Y, et al: miR-186 regulation of Twistl and ovarian cancer sensitivity to cisplatin. Oncogene 35: 323-332, 2016.

13. Guo Z, Chen W, Dai G and Huang Y: Cordycepin suppresses the migration and invasion of human liver cancer cells by downregulating the expression of CXCR4. Int J Mol Med 45: 141-150, 2020.

14. Ho SY, Wu WS, Lin LC, Wu YH, Chiu HW, Yeh YL, Huang BM and Wang YJ: Cordycepin Enhances Radiosensitivity in Oral Squamous Carcinoma Cells by Inducing Autophagy and Apoptosis Through Cell Cycle Arrest. Int J Mol Sci 20: E5366, 2019.

15. Wei C, Yao X, Jiang Z, Wang Y, Zhang D, Chen X, Fan X, Xie C, Cheng J, Fu J, et al: Cordycepin Inhibits Drug-resistance Non-small Cell Lung Cancer Progression by Activating AMPK Signaling Pathway. Pharmacol Res 144: 79-89, 2019.

16. Khan MA and Tania M: Cordycepin in Anticancer Research: Molecular Mechanism of Therapeutic Effects. Curr Med Chem 27: 983-996, 2020

17. Yoon SY, Park SJ and Park YJ: The Anticancer Properties of Cordycepin and Their Underlying Mechanisms. Int J Mol Sci 19: E3027, 2018

18. Knapek KJ, Georges HM, Van Campen H, Bishop JV, Bielefeldt-Ohmann H, Smirnova NP and Hansen TR: Fetal Lymphoid Organ Immune Responses to Transient and Persistent Infection with Bovine Viral Diarrhea Virus. Viruses 12: E816, 2020.

19. Cheung ST, Huang DP, Hui AB, Lo KW, Ko CW, Tsang YS, Wong N, Whitney BM and Lee JC: Nasopharyngeal carcinoma cell line (C666-1) consistently harbouring Epstein-Barr virus. Int J Cancer 83: 121-126, 1999.

20. Chang MM, Hong SY, Yang SH, Wu CC, Wang CY and Huang BM: Anti-Cancer Effect of Cordycepin on FGF9-Induced Testicular Tumorigenesis. Int J Mol Sci 21: E8336, 2020.

21. Wang Z, Wu X, Liang YN, Wang L, Song ZX, Liu JL and Tang ZS: Cordycepin Induces Apoptosis and Inhibits Proliferation of Human Lung Cancer Cell Line H1975 via Inhibiting the Phosphorylation of EGFR. Molecules 21: E1267, 2016.

22. Chen Y, Yang SH, Hueng DY, Syu JP, Liao CC and Wu YC: Cordycepin induces apoptosis of C6 glioma cells through the adenosine $2 \mathrm{~A}$ receptor-p53-caspase-7-PARP pathway. Chem Biol Interact 216: 17-25, 2014.

23. Lee SY, Debnath T, Kim SK and Lim BO: Anti-cancer effect and apoptosis induction of cordycepin through DR3 pathway in the human colonic cancer cell HT-29. Food Chemical Toxicol 60: 439-447, 2013.

24. Tao X, Ning Y, Zhao X and Pan T: The effects of cordycepin on the cell proliferation, migration and apoptosis in human lung cancer cell lines A549 and NCI-H460. J Pharm Pharmacol 68: 901-911, 2016.

25. Li XY, Tao H, Jin C, Du ZY, Liao WF, Tang QJ and Ding K: Cordycepin inhibits pancreatic cancer cell growth in vitro and in vivo via targeting FGFR2 and blocking ERK signaling. Chin J Nat Med 18: 345-355, 2020

26. Chen YH, Wang JY, Pan BS, Mu YF, Lai MS, So EC, Wong TS and Huang BM: Cordycepin enhances cisplatin apoptotic effect through caspase/MAPK pathways in human head and neck tumor cells. OncoTargets Ther 6: 983-998, 2013.

27. Gao Y, Chen DL, Zhou M, Zheng ZS, He MF, Huang S, Liao XZ and Zhang JX: Cordycepin enhances the chemosensitivity of esophageal cancer cells to cisplatin by inducing the activation of AMPK and suppressing the AKT signaling pathway. Cell Death Dis 11: $866,2020$.

28. Clevers $\mathrm{H}$ and Nusse $\mathrm{R}: \mathrm{Wnt} / \beta$-catenin signaling and disease. Cell 149: 1192-1205, 2012. 
29. Krishnamurthy Nand Kurzrock R: Targeting the Wnt/beta-catenin pathway in cancer: Update on effectors and inhibitors. Cancer Treat Rev 62: 50-60, 2018.

30. Su NW, Wu SH, Chi CW, Liu CJ, Tsai TH and Chen YJ: Metronomic Cordycepin Therapy Prolongs Survival of Oral Cancer-Bearing Mice and Inhibits Epithelial-Mesenchymal Transition. Molecules 22: E629, 2017.
31. Liu T, Zhu G, Yan W, Lv Y, Wang X, Jin G, Cui M, Lin Z and Ren X: Cordycepin Inhibits Cancer Cell Proliferation and Angiogenesis through a DEK Interaction via ERK Signaling in Cholangiocarcinoma. J Pharmacol Exp Ther 373:279-289, 2020.

c) (i) (9) This work is licensed under a Creative Commons cc) Attribution-NonCommercial-NoDerivatives 4.0 International (CC BY-NC-ND 4.0) License. 